\title{
Performance Enhancements of Mass Selective Axial Ejection from a Linear Ion Trap
}

\author{
Mircea Guna and Thomas A. Biesenthal \\ MDS Analytical Technologies, Concord, Ontario, Canada
}

\begin{abstract}
Mass selective axial ejection (MSAE) of ions from a linear ion trap (LIT) takes advantage of the rf fringing fields at the end of the linear quadrupole to convert radial ion excitation into axial ejection. Ions gain radial amplitude via a mass selective resonance excitation process and are ejected axially over an electrostatic DC barrier. The extraction efficiency and resolution are determined by the length and shape of the extraction region in the vicinity of the exit aperture. In the work presented here, axial DC fields, created by auxiliary electrodes, were used to modify the shape of the trapping electrostatic fields along the quadrupole axis and to increase the density of the ions in the extraction region. Better confinement and ion cloud coherence increased the extraction efficiency and the spectral resolution. As a result, extraction efficiency can be increased by up to one order of magnitude at fast scan rates, i.e., 10 and $20 \mathrm{kTh} / \mathrm{s}$, and a factor of 2-3 at the slower scan speed of $1 \mathrm{kTh} / \mathrm{s}$. The length of the extraction region was also modified by application of a portion of the drive rf voltage to the end lens of the LIT. A comparison of the MSAE spectra at different scan rates and rf levels showed that extraction efficiencies increase at scan rates of $10 \mathrm{kTh} / \mathrm{s}$ or higher, with associated improvements in mass spectral peak widths. (J Am Soc Mass Spectrom 2009, 20, 1132-1140) () 2009 American Society for Mass Spectrometry
\end{abstract}

$\mathrm{T}$ The development of the mass selective axial ejection (MSAE) from a linear ion trap (LIT) has enabled the development of the hybrid LIT triple quadrupole (QTRAP) mass spectrometer [1-3]. In this device the second mass analyzing quadrupole serves a dual role, as a standard transmission $\mathrm{rf} / \mathrm{DC}$ mass filter and as a LIT. Linear ion traps have higher acceptance than traditional three-dimensional (3D) ion traps $[1,4$, 5], since there is no quadrupole field along the z-axis. High trapping efficiencies coupled with efficient axial ion extraction have resulted in significant performance improvements with respect to conventional tandem mass spectrometers [1]. Here ions are trapped radially by an rf quadrupole field and axially by DC potentials at the ends of the quadrupole array. Trapped ions are cooled through collisions with the background gas molecules and ejected axially in a mass-selective fashion by ramping the amplitude of the main $\mathrm{rf}$ drive, to bring ions of increasingly higher $\mathrm{m} / \mathrm{z}$ into resonance with a single-frequency dipolar auxiliary signal, applied between two opposing rods. In the fringing fields the radial ion motion is coupled to axial motion allowing the ions gain additional sufficient axial ion kinetic energy to overcome the repulsive DC exit barrier.

For a mass selective ejection device the extraction efficiency, resolution and the scan rate are very impor-

Address reprint requests to M. Guna, MDS Analytical Technologies, 71 Four Valley Dr., Concord, Ontario, L4K 4V8, Canada. E-mail: michael.guna@ sciex.com tant because these affect the sensitivity and throughput for the mass spectral acquisition. The current work describes several approaches to increase the spectral resolution and sensitivity at fast scan rates for MSAE from a low-pressure LIT. Auxiliary electrodes were used to move the trapped ions toward the extraction region to provide better spatial ion confinement before and during the ejection process leading to improved ion cloud coherence and resolution.

Hager and Londry previously showed that the exit fringing fields can be modified by the addition to the exit lens of a portion of the drive rf voltage that is applied to one pole pair [6-8]. The voltage was coupled through a capacitive circuit from the same pole, i.e., pair of opposite rods, to which the dipolar excitation signal was applied. That work was limited to relatively low rf voltages of about $15 \%$ of the drive voltage, which was found to be effective for improving mass spectral performance for scan speeds up to $4 \mathrm{kTh} / \mathrm{s}$.

In the present work, it is shown that a further increase in the amount of rf coupled to the exit lens increases the extraction efficiency and resolution at scan rates of $10 \mathrm{kTh} / \mathrm{s}$ and higher.

This paper will detail experimental measurements on the spectral sensitivity and resolution of MSAE as a function of scan rate for ions of $\mathrm{m} / \mathrm{z}$ ratio $<1000 \mathrm{Th}$. The data will show that both the extraction efficiency and resolution are improved at fast scan rates in comparison to the conventional mode of operation, when either or both of the above methods were used. 
(a)
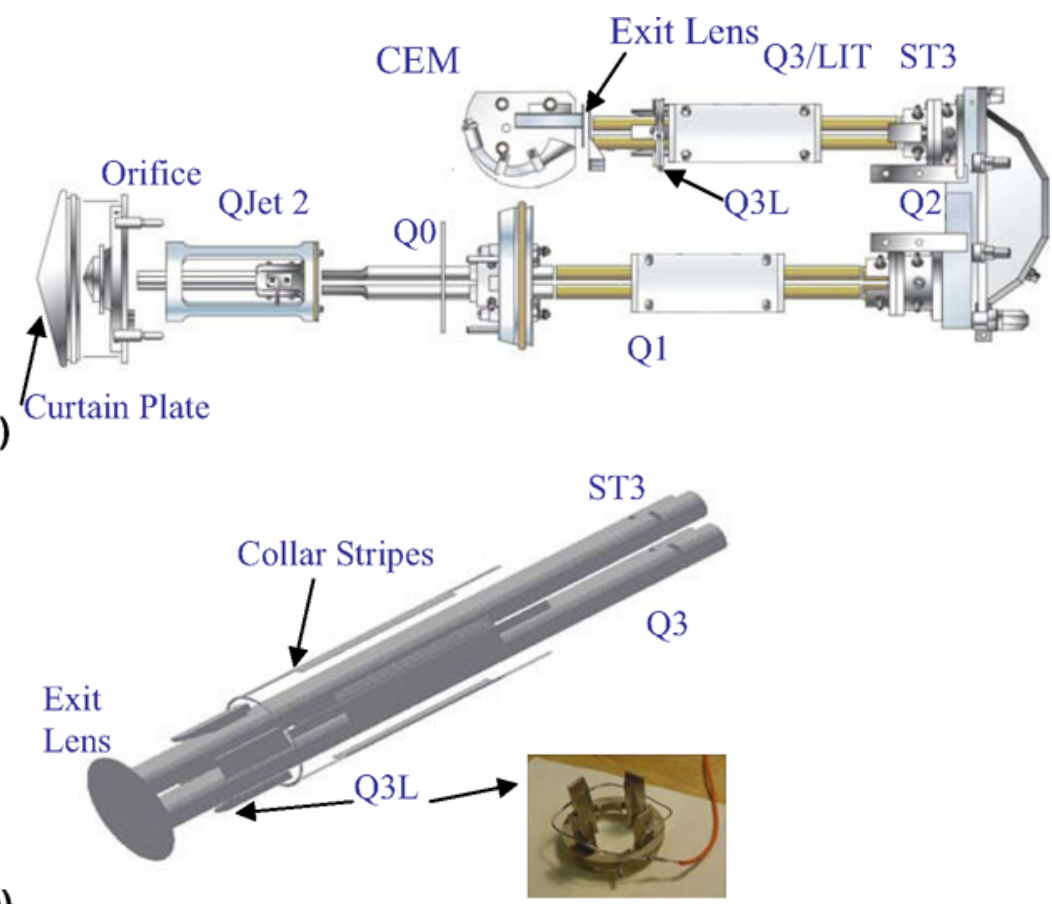

(b)

Figure 1. (a) A schematic of the Ion Path of the research-grade version QTRAP 5500 System. The linear ion trap is located in the Q3 position. (b) Rendered view of the linear ion trap and the auxiliary electrodes generated using the SIMION 7.0 software and a photograph showing the geometry of the auxiliary electrodes Q3L.

Simulations are presented to better understand how the modified fields at the end of the LIT change the ejection dynamics.

\section{Experimental}

Experiments were carried out on a research-grade version of the QTRAP 5500 System (AB/MDS Analytical Technologies, Concord, Ontario, Canada). A schematic of the instrument ion path is shown in Figure 1a. Ions were formed using an electrospray ionization source. The ions were transported through an aperture in a curtain plate, a $0.62 \mathrm{~mm}$ diameter sampling orifice and into a $12.5 \mathrm{~cm}$ long rf-only quadrupole ion guide (QJet 2) with a field radius of $2.0 \mathrm{~mm}$, operated at a drive frequency of $1.15 \mathrm{MHz}$. The pressure in the QJet 2 region was 3.5 Torr. Ions exiting the QJet 2 ion guide passed through a differential pumping aperture into an rf-only quadrupole (Q0) operated at $7 \mathrm{mTorr}$ of nitrogen. Nitrogen was used as a curtain gas. Ions exiting the Q0 quadrupole passed through a differential pumping aperture and into the first mass analyzing quadrupole (Q1). Ions were mass selected in Q1 and transferred at low energies, i.e., $10 \mathrm{eV}$, through a nitrogen filled collision cell (Q2), operated at a pressure of $\sim 10$ mTorr, into the Q3 linear ion trap. Two additional lenses were located downstream of Q3, the first a mesh covered 8 $\mathrm{mm}$ aperture, the second an $8 \mathrm{~mm}$ diameter aperture.
The mesh covered lens is referred to as the exit lens throughout this paper.

A portion of the main $\mathrm{rf}$ drive applied to the Q3 A-pole pair, e.g., the same set of opposite rods to which the dipolar excitation signal was applied, was added on top of the DC bias applied to the exit lens through a capacitive dividing network, Supplemental Figure S1, which can be found in the electronic version of this article. Previous experiments showed that the MSAE performance was very poor when the rf voltage was coupled to the exit lens from the opposite pole, i.e., the B-pole [6-8]. Therefore, in all the experiments described here the rf on the exit lens was coupled from the A-pole.

Three different configurations were chosen: $0 \%, 25 \%$, and $50 \%$ of the drive rf voltage. Supplemental Figure S1 shows the two rf voltage dividing networks used here. The actual rf coupling ratios were lower in reality due to parasitic capacitance created by the detector and the surrounding ground electrodes, thus for the $25 \%$ setup it was estimated that the voltage applied to the exit lens was only about $15 \%$, while for the $50 \%$ setup the voltage applied for the exit lens was at most $30 \%$.

In these experiments the Q1 quadrupole was used in mass analyzing mode at open resolution, i.e., the whole isotopic cluster was passed through, while Q3 was exclusively used as a linear ion trap. Ions were trapped in the LIT by raising the DC potentials on the exit lens and a short rf-only quadrupole (ST3) located between 
the Q2 collision cell and the Q3 quadrupole. Ions were then concentrated in the extraction region by applying a DC voltage to the auxiliary electrodes interposed between the quadrupoles rods. Ions were scanned out of the LIT at rates of 1 to $20 \mathrm{kTh} / \mathrm{s}$ using the technique of MSAE. In the present work ion excitation was achieved via resonance excitation with a single frequency dipolar auxiliary signal applied between two opposing rods, e.g., A-pole pair, using a bipolar toroidal transformer (Supplemental Figure S1). Ion ejection was carried out at a frequency of excitation of $470 \mathrm{kHz}$ corresponding to a Mathieu stability parameter of $\mathrm{q}=0.846$ at a drive frequency of $1.228 \mathrm{MHz}$. All excitation amplitudes reported in the paper were measured zero to peak, pole to pole. In the trap scan mode the pressure in the analyzer region was maintained at 0.04 mTorr of nitrogen derived from both the curtain gas and primarily the Q2 collision cell.

The Q3 auxiliary electrodes consisted of a set of four electrodes $2.5 \mathrm{~cm}$ long, that had T-shaped cross section, interposed between the main quadrupole rods. The four auxiliary electrodes were held at the same DC potential but the depth of the stem section varied to enable the generation of an axial potential wall that was higher at the end of the mounting ceramic collar, i.e., $5 \mathrm{~cm}$ from the end of the quadrupole rod array, and shallower toward the center of the extraction region of the trap, Figure $1 \mathrm{~b}$ and $\mathrm{c}$.

The scan sequence consisted of an accumulation, cooling, mass scanning, and emptying periods. During the accumulation/fill step the DC voltage on the exit lens was set to $28 \mathrm{~V}$ repulsive relative to the DC bias of the Q3 linear ion trap. At the end of accumulation period the DC voltage on the short rf-only Brubaker quadrupole ST3 was set to a high repulsive value relative to the DC bias of the LIT and the ions were allowed to cool. The interior of the mounting ceramic collar of the LIT had conductive metal stripes. During the measurement sequence the voltage on these stripes was set at $1500 \mathrm{~V}$ [9]. The voltage on the Q3 auxiliary electrodes was set at $0 \mathrm{~V}$ during the fill time and most of the cool time and then raised to $300 \mathrm{~V} 10 \mathrm{~ms}$ before the scan. During the cooling period the DC bias of the LIT was stepwise dropped to the scan value $-160 \mathrm{~V}$. Just before the mass scan the DC voltage on the exit lens voltage, EXB, was dropped to the scan value, i.e., 3 to 40 $\mathrm{V}$ repulsive with respect to the DC bias of the Q3 LIT depending on the mass of the ions and the scan rate. The drive rf and auxiliary AC voltages were ramped over the chosen $\mathrm{m} / \mathrm{z}$ range to extract the ions from the LIT. Finally, during the emptying period, the trapping rf and DC voltages were lowered sufficiently to eject any residual trapped ions. Ions were detected by a Burle Industries (Lancaster, PA) channel electron multiplier, CEM, operated in pulse counting mode, with the entrance voltage floated to $-6 \mathrm{kV}$.

The analytes were from a 1:100 dilution of the electrospray calibrant solution (G2421A) obtained from Agilent Technologies Canada (Mississauga, ON). The dilution solution was 95:5 acetonitrile/water. The solution was infused at a rate of $7 \mu \mathrm{L} / \mathrm{min}$.

\section{Geometry of the Auxiliary Electrodes and the Potential Field Simulations}

SIMION 7.0 (Idaho National Laboratory) ion trajectory simulator was used to determine the potential along the axis of the linear quadrupole constructed of round rods in the presence of both the T-shaped Q3 auxiliary electrodes (Q3L) and the silver painted ceramic collar as well as to study the ions dynamics during the LIT scan. Figure 2a shows the electrostatic potential field along the axis of the quadrupole during the fill time when the DC bias of the quadrupole ion trap was set at $-28 \mathrm{~V}$. Due to the small potential difference between the LIT DC bias and the Q3L bias the Q3L auxiliary electrodes did not play a role in the ion population dynamics during the fill time. On the other hand, the $1500 \mathrm{~V}$ applied to the painted stripes within the ceramic mounting collar forced the ion population to split into

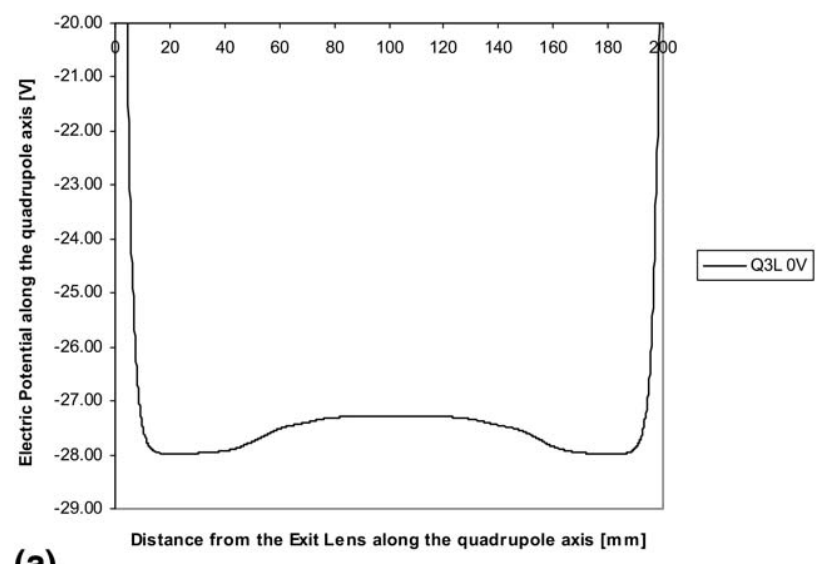

(a)

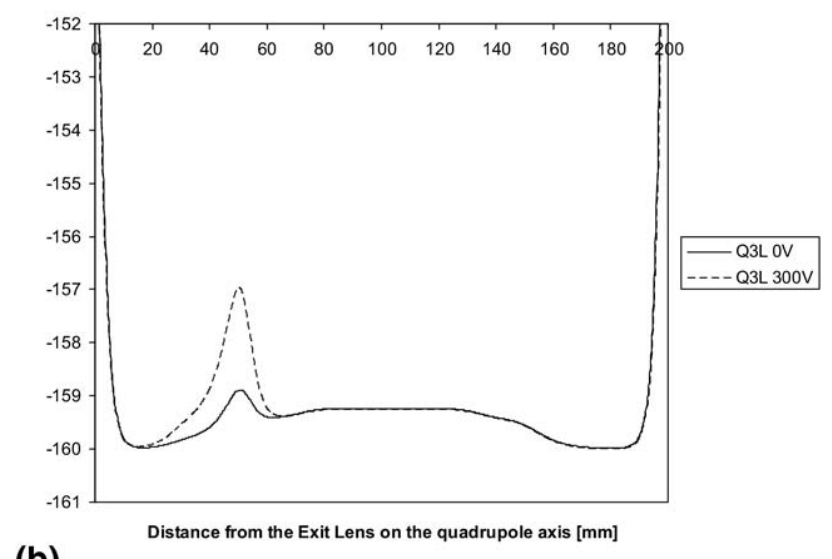
(b)

Figure 2. Plots of the axial electrostatic potential along the quadrupole axis. (a) During the LIT fill time when the Q3L voltage was 0 $\mathrm{V}$, the LIT DC rod offset voltage was $-28 \mathrm{~V}$ and the voltage applied to the silver painted stripes was $1500 \mathrm{~V}$; (b) $10 \mathrm{~ms}$ before the scan and during the scan when LIT DC rod offset voltage was $-160 \mathrm{~V}$, the voltage applied to the silver painted stripes was $1500 \mathrm{~V}$, and the Q3L voltage was 0 and $300 \mathrm{~V}$, respectively. 
two groups of ions separated by the dimensions of the collar (i.e., $10 \mathrm{~cm}$ long). Judging by the symmetry of the electrostatic field along the axis it can be inferred that about $50 \%$ of the ions trapped near the exit lens were available during the analytical scan. The remaining trapped ions would either be lost on the quadrupole electrodes during the trap scan, or dumped during the emptying step. The continuous trace in Figure $2 \mathrm{~b}$ shows the potential along the quadrupole axis during the cool time, when the DC bias of the LIT was set to the scan value, i.e., $-160 \mathrm{~V}$, and the voltage on the auxiliary electrodes was $0 \mathrm{~V}$. The dotted trace in Figure $2 \mathrm{~b}$ represents the potential along the quadrupole axis $10 \mathrm{~ms}$ before and during the scan when the voltage on the auxiliary electrodes was raised to $300 \mathrm{~V}$. One can see that the voltage on the Q3L auxiliary electrodes now plays a role in the shape of the DC potential walls of the extraction region of the LIT. Ion trajectory simulations showed that the ions, once cooled, tend to slowly move toward the exit lens, before the scan, due to the steep potential gradient

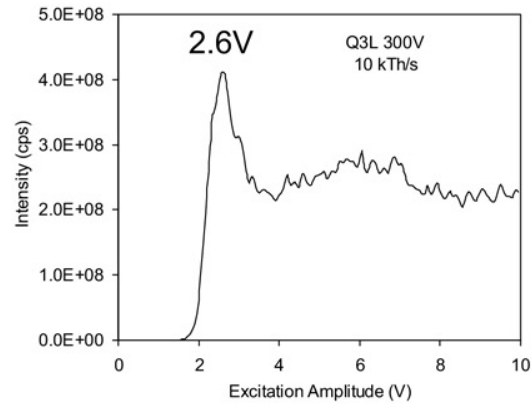

(a)

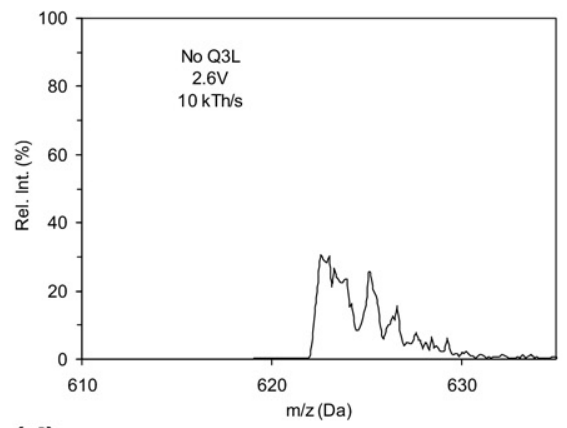

(d)

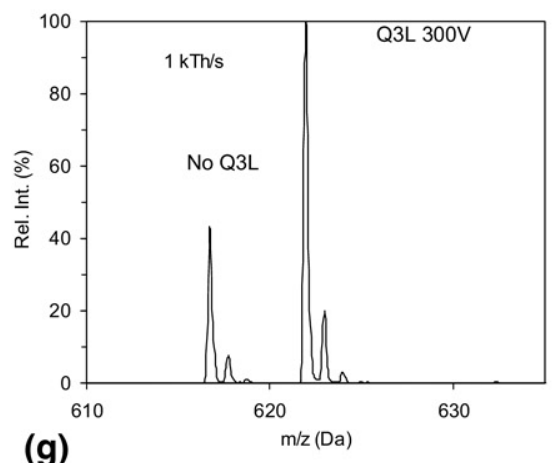

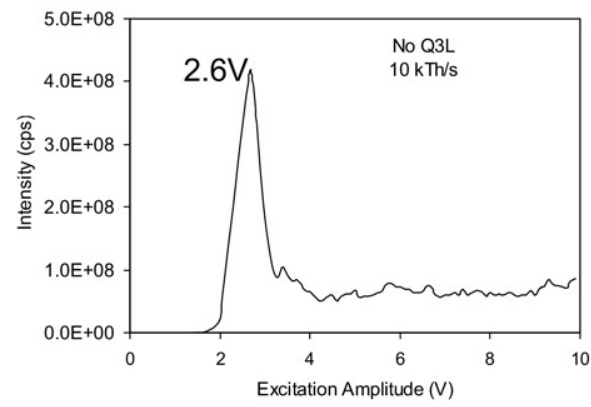

(b)

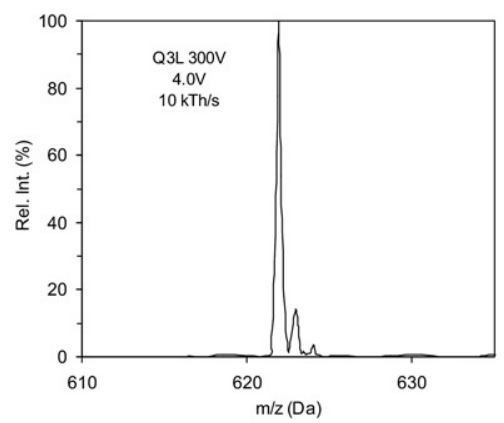

(e)

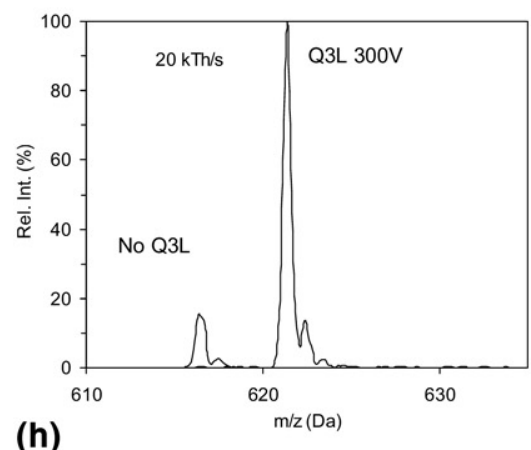

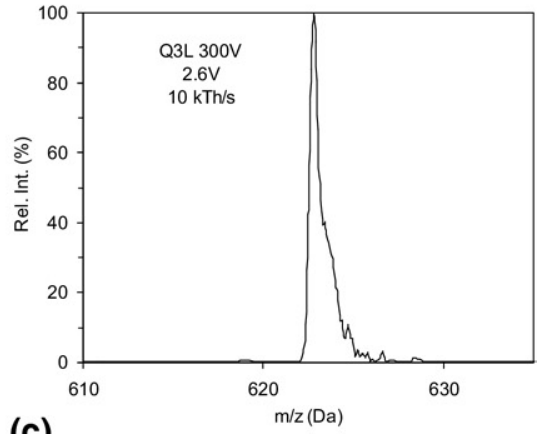

(c)

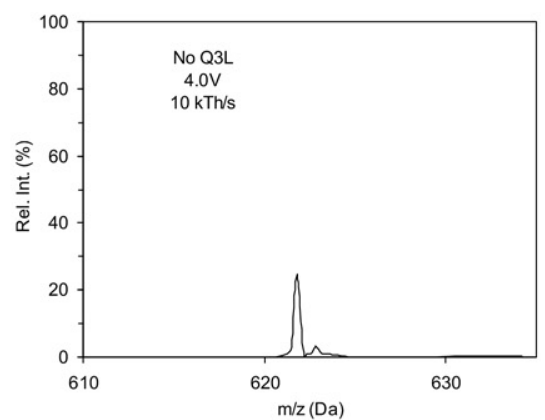

(f)

Figure 3. Experimental data comparison of the LIT spectra obtained in both the presence and absence of the Q3L auxiliary electrodes. (a) Total ion chromatogram (TIC) of 10 MCA LIT spectra acquired at $10 \mathrm{kTh} / \mathrm{s}$. The Q3L voltage was $300 \mathrm{~V}$. (b) Total ion chromatogram (TIC) of 10 MCA LIT spectra acquired at $10 \mathrm{kTh} / \mathrm{s}$. No Q3L. (c) and (d) LIT spectra at scan rate $10 \mathrm{kTh} / \mathrm{s}$ and excitation amplitude 2.6 V with and without Q3L, respectively. Data were normalized to the intensity of the peak acquired in the presence of Q3L. (e) and (f) LIT spectra at scan rate $10 \mathrm{kTh} / \mathrm{s}$ and excitation amplitude $4.0 \mathrm{~V}$ with and without Q3L, respectively. Data were normalized to the intensity of the peak acquired in the presence of Q3L. (g) LIT spectra at scan rate $1 \mathrm{kTh} / \mathrm{s}$ with and without Q3L. The spectra acquired in the absence of the Q3L electrodes were shifted for better visualization. Data were normalized to the intensity of the peak acquired in the presence of Q3L. (h) LIT spectra at scan rate $20 \mathrm{kTh} / \mathrm{s}$ with and without Q3L. The spectra acquired in the absence of the Q3L electrodes were shifted for better visualization. Data were normalized to the intensity of the peak acquired in the presence of Q3L. 
created by the Q3L electrodes. As discussed in the following section this improves the ion cloud coherence and increases the MSAE extraction efficiency.

\section{Results and Discussion}

\section{Extraction Efficiency and Peak Resolution. Q3L Auxiliary Electrodes}

Figure $3 a$ and $b$ show the total ion signal as a function of the auxiliary excitation AC voltage for MSAE of the isotopic cluster near $\mathrm{m} / \mathrm{z} 622$, at a scan rate of $10 \mathrm{kTh} / \mathrm{s}$ in two different configurations: 3a, Q3L was installed and the voltage applied to the electrodes was $300 \mathrm{~V}$ and $3 \mathrm{~b}$, no Q3L auxiliary electrodes were installed. The excitation voltage was ramped in steps of $0.1 \mathrm{~V}$ and each spectrum was a sum of 10 scans. The rf coupled to the exit lens was $30 \%$. The most obvious difference between the two figures was the much more dramatic drop of intensity with increased auxiliary excitation AC voltage in the absence of the Q3 LIT auxiliary electrodes versus intensity in the Q3L configuration. Figure $3 c$ and $\mathrm{d}, 3 \mathrm{e}$ and $\mathrm{f}$, show the mass spectra acquired with both configurations when the excitation amplitude was 2.6 and $4 \mathrm{~V}$, respectively. At $2.6 \mathrm{~V}$, in the absence of the Q3 LIT auxiliary electrodes, the ions exited the trap in several packets at different times and rf levels, Figure $3 \mathrm{~d}$. While the sensitivity was the highest at an auxiliary AC excitation amplitude of $2.6 \mathrm{~V}$ the observed peak resolution was very poor. When the excitation amplitude was increased to $4.0 \mathrm{~V}$, the peak shape and resolution improved for both configurations but the ion intensities dropped, Figure 3e and f. At low AC amplitudes, there is insufficient energy to drive the ions to the rods promptly. Since the ions have a much greater axial extent without the Q3L electrodes their ejection times are spread out concomitantly. The greater intensity decrease without the electrodes has a similar explanation in that when there is sufficient energy in the auxiliary AC field to produce prompt ejection, there is also sufficient energy to drive the ions to the rods. If the ions are axially diffuse, there is then a greater opportunity for them to be driven to the rods and neutralized. In the presence of the auxiliary electrodes the ion cloud is confined in the vicinity of the extraction region and the ions exit the trap more coherent in time, thus the improved spectral resolution.

Figure $3 \mathrm{~g}$ and $\mathrm{h}$ show a comparison of the LIT spectra of the $\mathrm{m} / \mathrm{z} 622$ isotopic cluster with and without the Q3L auxiliary electrodes at scan rates 1 and 20 $\mathrm{kTh} / \mathrm{s}$. The gain in sensitivity and resolution is greater at the higher scan rates. This is explained by the fact that the magnitude of the optimum auxiliary AC excitation amplitude is a strong function of scan rate. The larger auxiliary AC amplitudes result in more rapid acceleration in the radial dimension.

\section{Spectral Sensitivity and Resolution Versus rf} Coupling on the Exit Lens. Experimental Data

Figure 4 displays the trapped ion mass spectra of three different analyte ions with $\mathrm{m} / \mathrm{z}$ ratios 322, 622, and 922 Th, at different scan speeds and levels of rf coupling to the exit lens. The LIT was tuned to reach a peak full width at half height $(\mathrm{FWHH})$ of $<0.25 \mathrm{Th}$ at $1 \mathrm{kTh} / \mathrm{s}$, $<0.4 \mathrm{Th}$ at $10 \mathrm{kTh} / \mathrm{s}$, and $<0.7 \mathrm{Th}$ at $20 \mathrm{kTh} / \mathrm{s}$. The spectra were artificially shifted on the $\mathrm{m} / \mathrm{z}$ scale to aide comparisons. The addition of the rf voltage to the exit lens improved the mass spectral performance dramatically at $1 \mathrm{kTh} / \mathrm{s}$. However, little difference in performance was observed when the rf percentage was increased furthermore from $15 \%$ to $30 \%$., Figure $4 a-c$.

Performance at higher scan rates is much more dependent upon the amount of $\mathrm{rf}$ applied to the exit lens. The LIT spectra acquired at scan rates of 10 and $20 \mathrm{kTh} / \mathrm{s}$, as shown in Figure $4 \mathrm{~d}-\mathrm{f}$ and $4 \mathrm{~g}-\mathrm{i}$, respectively, illustrate that a further increase in signal intensity is achieved as the amount of rf voltage is increased from $15 \%$ and to $30 \%$. There is not a strong mass-dependency of the performance enhancements at the two faster scan rates displayed in Figure 4. The improvements in signal intensity and peak shape are about the same at $\mathrm{m} / \mathrm{z} 322$ and 922 .

\section{Sensitivity and Resolution Improvements. Comparison Between the Standard Configuration and the New Set-Up: High rf Coupling and Auxiliary T-Electrodes}

Overall the sensitivity improvements observed when higher $\mathrm{rf}$ levels were applied to the exit lens and the auxiliary Q3 electrodes are installed, were about 2- to 4 -fold at $1 \mathrm{kTh} / \mathrm{s}$ while at the higher scan speeds, 10 and $20 \mathrm{kTh} / \mathrm{s}$, the gains were up to an order of magnitude.

The resolution also improved, thus at $10 \mathrm{kTh} / \mathrm{s}$ the FWHH was $<0.4$ Th versus $0.65 \mathrm{Th}$ in the standard set-up while at $20 \mathrm{kTh} / \mathrm{s}$ the FWHH improved to about 0.6 Th from $0.85 \mathrm{Th}$.

\section{Ion Trajectory Simulation}

The addition of properly phased rf to the exit lens changes the dynamics of the ion motion in the extraction region and increases the coupling of the radial motion to the axial motion [7, 8]. These effects were studied by recording the ion velocity, position, and instantaneous electric field along the trajectory of the ions during the trap scan, using the SIMION 7.0 ion trajectory simulator. A 3D model of the LIT was created and the rf voltage was ramped toward higher amplitudes at $10 \mathrm{kTh} / \mathrm{s}$ while the excitation voltage was applied in a dipolar manner on a set of two opposing electrodes at an amplitude of $3 \mathrm{~V}$. The Q3L voltage was set to $300 \mathrm{~V}$ and the DC barrier at the exit lens was $15 \mathrm{~V}$. Three different rf coupling levels were 


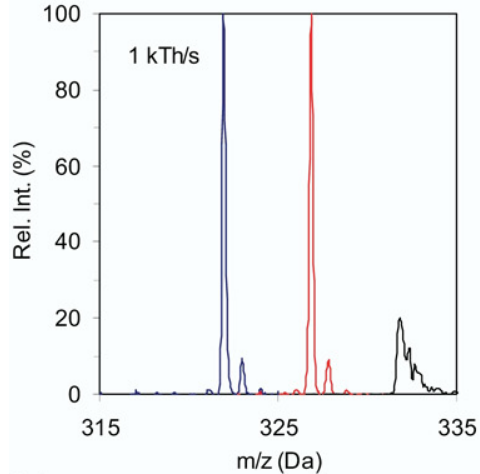

(a)

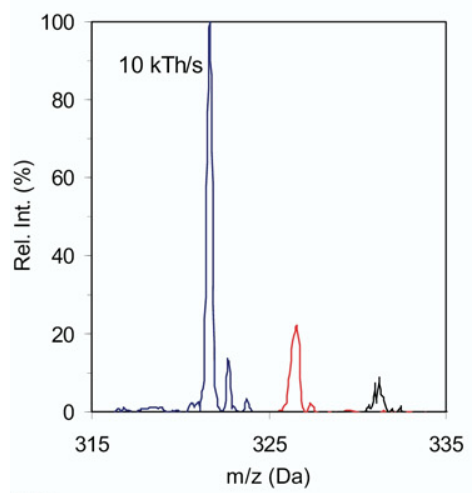

(d)

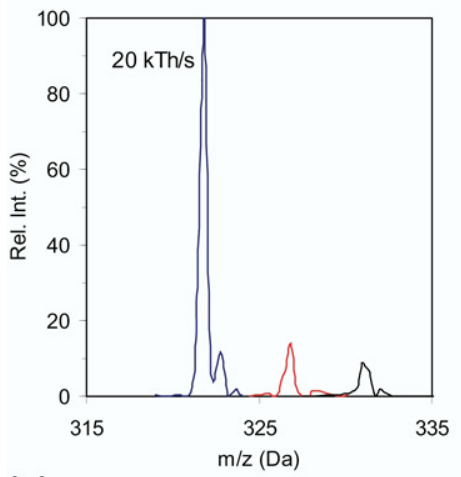

(g)

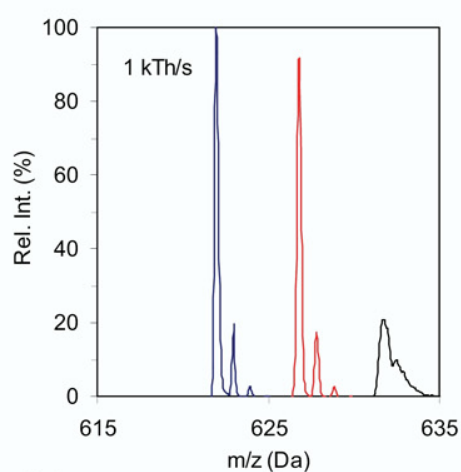

(b)

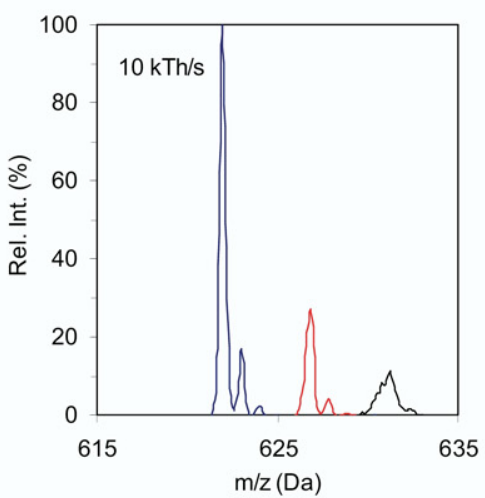

(e)

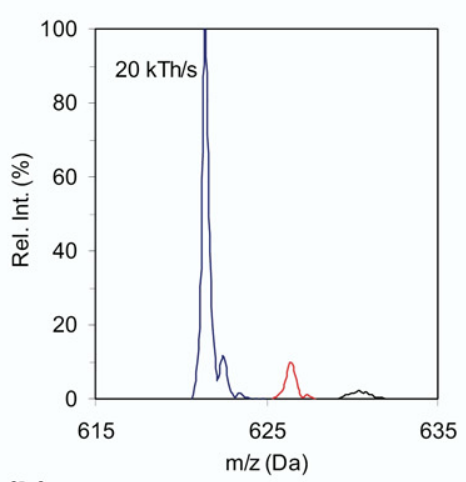

(h)

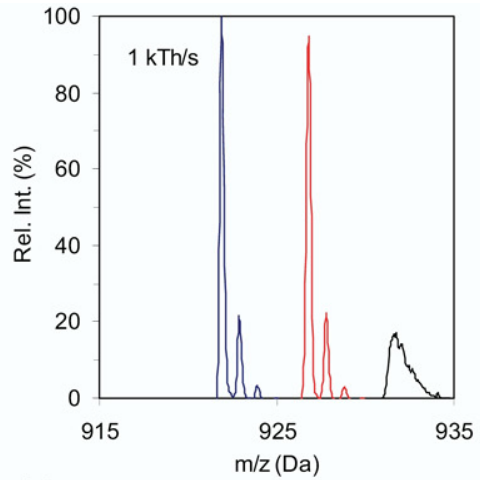

(c)

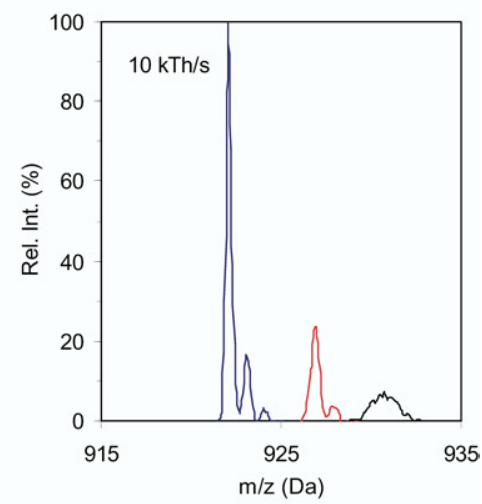

(f)

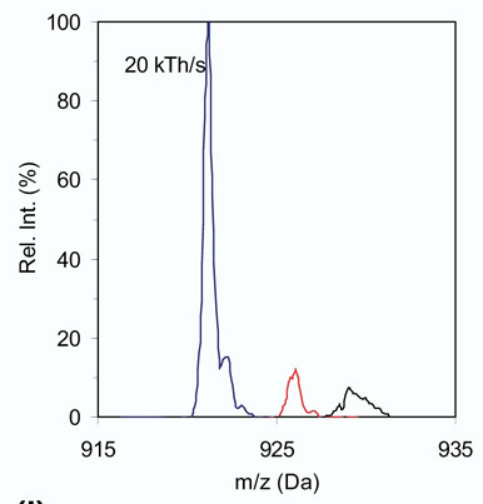

(i)

Figure 4. LIT Spectra acquired at different rf coupling: black, red, and blue traces correspond to $0 \%$, $15 \%$, and $30 \%$, respectively. (a)-(c) LIT spectra of ions of mass-to-charge ratio 322, 622, and 922 Th, respectively. Scan rate was $1 \mathrm{kTh} / \mathrm{s}$. (d)-(f) LIT spectra of ions of mass-to-charge ratio 322, 622, and $922 \mathrm{Th}$, respectively. Scan rate was $10 \mathrm{kTh} / \mathrm{s}$. (g)-(i) LIT spectra of ions of mass-to-charge ratio 322, 622, and $922 \mathrm{Th}$, respectively. Scan rate was $20 \mathrm{kTh} / \mathrm{s}$. Data in each figure were normalized to the highest intensity peak for that specific ion and scan rate.

simulated: no rf, low level $(15 \%)$, and high level $(30 \%)$. No collisions with the ambient gas were considered. The potential array (PA) file was updated every $0.2 \mu \mathrm{s}$. Ion ejection was carried out at a frequency of excitation of $380 \mathrm{kHz}$ corresponding to a Mathieu stability parameter of $\mathrm{q}=0.846$ at a drive frequency of $1 \mathrm{MHz}$.

Figure 5 a shows the ion trajectories for ions ejected using MSAE at three different rf levels. It was observed, when analyzing the variation in time of the ions' velocity in y direction (Figure 5b), that at an excitation amplitude of $3 \mathrm{~V}$ the ions start to gain radial energy when the main rf level corresponds to $\sim 10$ Th (at 10 $\mathrm{kTh} / \mathrm{s} 1 \mathrm{Th}$ is ramped in $100 \mu \mathrm{s}$ ) lower than the mass of the ions. This is due to the broadening of the absorption spectra at large excitation amplitudes [10]. Due to the great amount of data generated by the simulator the start mass of the scan was chosen 10 Th lower than the mass (622 Th) of the analyte ion. From Figure 5a it can be concluded that at a barrier of $15 \mathrm{~V}$ the ions gain 


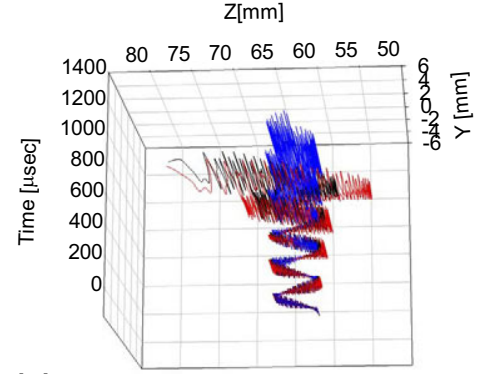

(a)

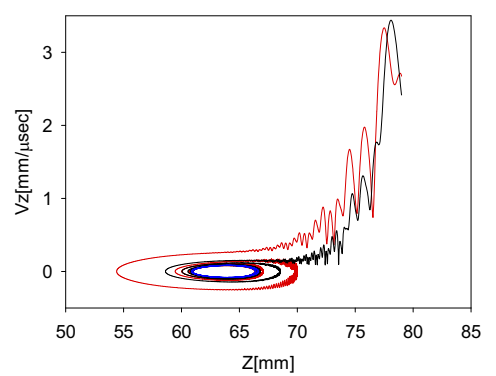

(d)

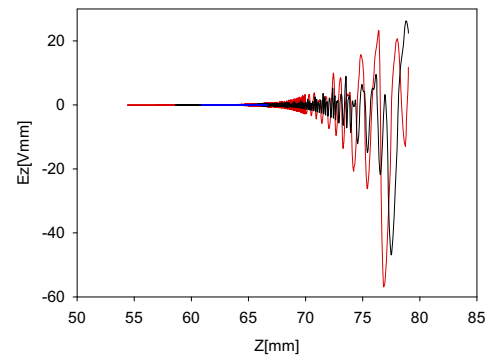

(g)

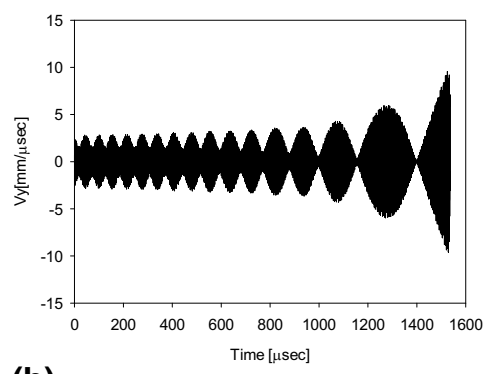

(b)

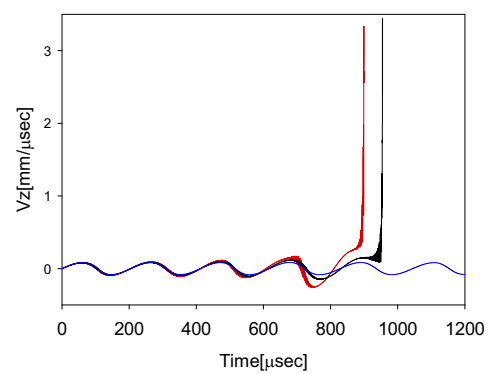

(e)

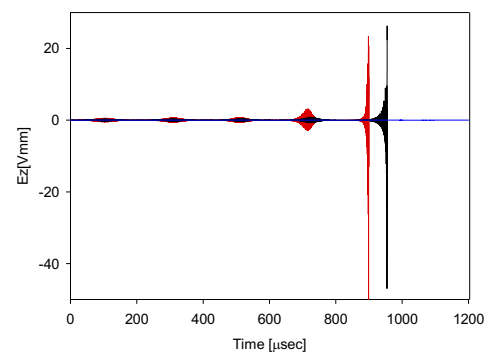

(h)

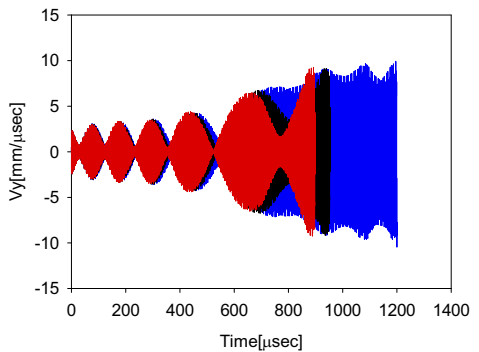

(c)

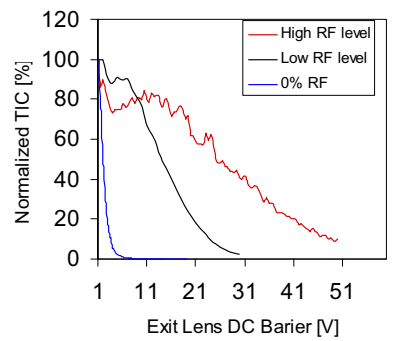

(f)

\begin{abstract}
Figure 5. Ion dynamics during the LIT scan. Trajectories were simulated for ions of the same mass at different rf levels applied to the exit lens. Q3L at $300 \mathrm{~V}$. Red, black, and blue trajectories were recorded when the rf applied to the exit lens was 30\%,15\%, and $0 \%$ respectively. (a) The trajectory of the ions in the $y$ and $z$ directions versus time. The exit lens plane is at $z=80 \mathrm{~mm}$. $Y$ is the plane of excitation. $Z$ axis is the quadrupole axis. (b) The velocity of an ion along the $y$-axis versus time when the trap was scanned starting 15 Th before the mass of the analyte. (c) The velocity of the ions along the y-axis versus time when the trap was scanned starting 10 Th before the mass of the analyte. (d) The velocity of the ions along the $\mathrm{z}$-axis versus ion position on $\mathrm{z}$-axis. At higher $\mathrm{rf}$ on the exit lens the ions gain more axial kinetic energy than at lower rf levels, thus travel deeper inside the ion trap potential walls, both toward the Q3L electrodes, at lower z, and toward the exit lens at higher z. (e) The velocity of the ions along the $\mathrm{z}$-axis versus time. Ions gain axial energy at a faster rate when the rf coupling was higher. (f) TIC experimental data acquired at $10 \mathrm{kTh} / \mathrm{s}$ and different $\mathrm{rf}$ ratios applied on the exit lens. The DC barrier applied to the exit lens was ramped with steps of $0.5 \mathrm{~V}$. $(\mathrm{g})$ The momentary axial electric field experienced by the ions versus ion position on the $\mathrm{z}$-axis. (h) The momentary axial electric field experienced by the ions versus time.
\end{abstract}

enough axial energy to overcome the DC barrier and get ejected only when the rf is coupled to the exit lens, exit lens plane being situated at $Z=80$. When no $\mathrm{rf}$ is applied to the exit lens the amplitude of the ion motion in the y direction (yz-plane of excitation) increases until it exceeds the internal radius of the rod array, i.e., 4.17 $\mathrm{mm}$ and the ion ends up being lost on the rods. Despite the fact that the energy gained in the y direction is similar for all cases, the energy that is coupled in the axial direction varies with the amount of rf coupling (Figure $5 \mathrm{~d}$ and e), i.e., the higher the $\mathrm{rf}$ on the exit lens the higher the energy accumulation rate in the axial direction. The ions travel deeper inside the trap (Figure $5 \mathrm{a}, \mathrm{d})$, up the electrostatic potential wall created by the voltage applied to the exit lens and Q3L electrodes, and each time they come into the vicinity of the fringing fields they gain more axial energy until the energy is high enough to overcome the exit barrier and the ion is ejected out of the trap. This is because both the magnitude and the degree of penetration of the axial fringing field are higher at higher rf coupling (Figure $5 \mathrm{~g}$ and $\mathrm{h}$ ). Experimental results (Figure 5f) confirmed the fact that more energy was coupled axially at high rf coupling. In these experiments the trap was scanned at $10 \mathrm{kTh} / \mathrm{s}$ and 
the total ion chromatogram (TIC) was recorded while increasing the repulsive DC potential applied to the exit lens in $0.5 \mathrm{~V}$ steps. A comparison of the TIC curves show that the extraction efficiency versus the exit barrier decreases more rapidly at low levels of rf coupling than at high rf coupling with increasing exit barrier. At a high rf level on the exit lens, 10\%, 40\%, and $90 \%$ of the ions have axial kinetic energies in excess of 50,30 , and $10 \mathrm{eV}$, respectively. At a low rf level only $2 \%$ of the ions have axial kinetic energies higher than $30 \mathrm{eV}$ and $70 \%$ higher than $10 \mathrm{eV}$. At no rf on the exit lens the energy of the ions does not exceed $5 \mathrm{~V}$.

The ability to tune the trap at higher exit barriers has an influence on the spectral resolution and sensitivity of the MSAE spectra. Figure 6 shows the influence of the exit barrier on the sensitivity of the resolution of the MSAE spectra of the isotope cluster of the 622 Th ion, when the rf coupling was 30\%, Q3L voltage was $300 \mathrm{~V}$ and the excitation amplitude was $3.9 \mathrm{~V}$. The exit barrier voltage was ramped up in $0.5 \mathrm{~V}$ steps.

At low exit barriers, i.e., approximately $6 \mathrm{~V}$, the sensitivity was relatively high but peak resolution was poor. When the barrier was increased to $15 \mathrm{~V}$ the total ion count (TIC) drops approximately $10 \%$ but the peak height increased and the resolution improved. Ions are forced to gain more axial energy to overcome the higher barrier and oscillate a bit longer in the trap; this results in a mass shift towards higher $m / z$ ratios, however the mass selective characteristic of the radial-to-axial energy conversion improves the temporal coherence of the ions and both the peak intensity and resolution increase.

When the exit barrier is increased to $24 \mathrm{~V}$ the sensitivity drops furthermore by at least factor of two. During the MSAE process ions gain both axial and radial kinetic energy, Figure $5 c$ and $d$. The increase in the exit barrier forces the ions to gain significantly more axial energy to be axially ejected, meanwhile their radial energy as well as their displacement in the y direction increases, Figure 5a-c. If their radial energy exceeds the rf trapping potential, the ions are lost on the rods. The ratio between the axially ejected and those lost on the rods decreases with increased exit barrier.

In practice, the exit lens barrier is tuned to achieve the best spectral peak resolution with a minimum loss in sensitivity. Higher rf coupling increases the range of the exit barrier values over which both the sensitivity and resolution are high, improving the ease-of-tuning of the instrument. At low rf coupling and with no rf coupled to the exit lens, the tuning window becomes significantly narrower and the loss in sensitivity required to achieve good resolution is high.
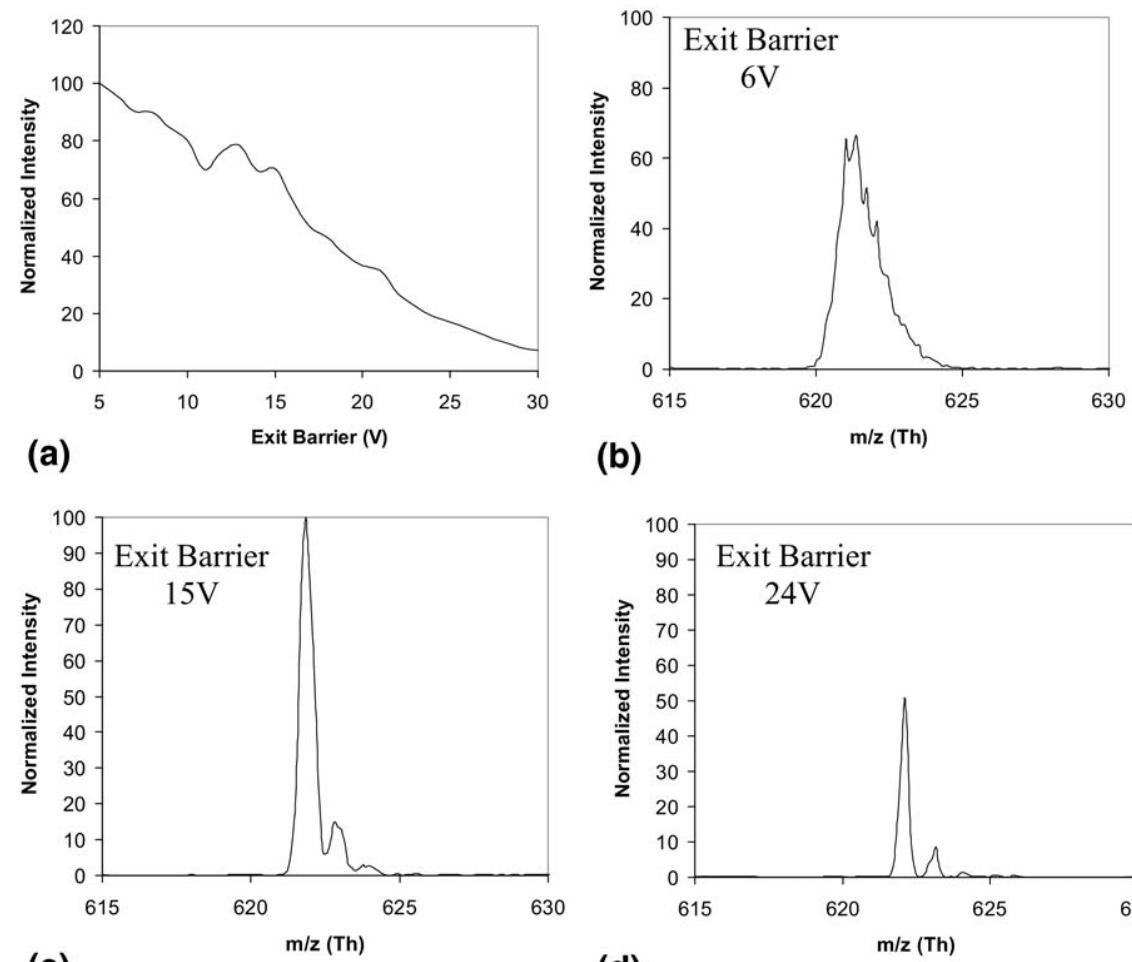

(b)

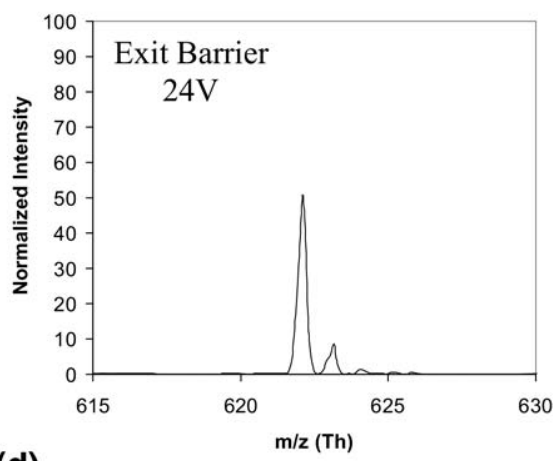

(d)

Figure 6. Influence of the exit barrier on the MSAE spectral resolution and sensitivity. Experimental LIT data acquired at $10 \mathrm{kTh} / \mathrm{s}$ when Q3L voltage was $300 \mathrm{~V}$, the rf coupling was $30 \%$, and the excitation amplitude used was $3.9 \mathrm{~V}$. LIT spectra were normalized to the highest peak, i.e., the spectra acquired at $15 \mathrm{~V}$ exit barrier. (a) TIC versus exit barrier. (b) LIT spectra at $6 \mathrm{~V}$ exit barrier. (c) LIT spectra at $15 \mathrm{~V}$ exit barrier. (d) LIT spectra at $24 \mathrm{~V}$ exit barrier. 


\section{Conclusions}

It has been shown that the addition of auxiliary DC fields within the linear ion trap and modification of the shape of the fringing fields changes the dynamics of the ion motion during the linear ion trap scan. Improved confinement provided by the axial DC fields increases the ion cloud coherence and reduces the ion losses on the rods during the scan. Application of a portion of the drive rf voltage on the exit lens improves the coupling between the axial and radial motion of the ions. Ions gain axial energy at a faster rate and are ejected with greater peak resolution and sensitivity, especially at fast scan speeds. The improvements observed vary with scan rate. The higher the scan rate the greater the improvements in extraction efficiency that were observed.

\section{Acknowledgments}

The authors thank Dr. Jim Hager for critical reading of this manuscript and thoughtful suggestions before submission. They thank Dr. Bruce Collings for his support and many helpful discussions regarding this work and gratefully acknowledge the tremendous support of the engineering team during the research and development of the QTRAP 5500 System.

\section{Appendix A Supplementary Material}

Supplementary material associated with this article may be found in the online version at doi:10.1016/ j.jasms.2009.02.002.

\section{References}

1. Hager, J. W. A New Linear Ion Trap Mass Spectrometer. Rapid Commun. Mass Spectrom. 2002, 16, 512-526.

2. Londry, F. A. Mass-Selective Axial Ejection from a Linear Quadrupole Ion Trap. Proceedings of the 50th ASMS Conference; Orlando, FL, June 2002; Poster MPM 377.

3. Londry, F. A.; Hager, J. W. Mass Selective Axial Ion Ejection from a Linear Quadrupole Ion Trap. J. Am. Soc. Mass Spectrom. 2003, 14, $1130-1147$

4. Schwartz, J. C.; Senko, M. W.; Syka, J. E. P. A Two-Dimensional Ion Trap Mass Spectrometer. J. Am. Soc. Mass Spectrom. 2002, 13, 659-669.

5. Hashimoto, Y.; Hasegawa, H.; Baba, T.; Waki, I. Mass Selective Ejection by Axial Resonant Excitation from a Linear Ion Trap. J. Am. Soc. Mass Spectrom. 2006, 17, 685-690.

6. Hager, J. W.; Londry, F. A. U.S. patent 7019 290, 2006

7. March, R. E.; Todd, J. Practical Aspects of Trapped Ion Mass Spectrometry Vol IV. Chapter 12, Hager, J. W, Ed.; Taylor and Francis Publishers: 2009 (in press).

8. Londry, F. A. unpublished.

9. Londry, F. A.; Stott, W. A.; Collings, B. A.; Hager, J. W. U.S. patent 6909 089, 2005.

10. French, A. P. Vibrations and Waves; W. W Norton and Company, Inc.: New York; p. 189-196. 